\title{
Improving Point-of-care Testing with Automated Identification Technologies
}

a report by

\section{Anand S Dighe and Kent Lewandrowski}

\author{
Assistant Director, Core Laboratory, Department of Pathology, and Associate Director of
} Laboratory Medicine, Massachusetts General Hospital

DOI:10.17925/EE.2006.00.02.1d

Anand S Dighe is Assistant Director of the Core Laboratory in the Department of Pathology at the Massachusetts General Hospital and an instructor in pathology at Harvard Medical School. Dr Dighe specialises in applications of clinical informatics in pathology. His primary interest is in the research and development of Internet-based expert system software for pathology interpretations. Other interests include Web-based applications for clinical education and the

development of expert systems for laboratory test selection.

Kent Lewandrowski is Associate Director of Laboratory Medicine at Massachusetts General Hospital and Director of the point-of-care testing programme. He is also an associate professor at Harvard Medical School, with research interests in pancreatic pathology and the evaluation of point-of-care technologies.

Dr Lewandrowski's interest in pointof-care testing began while he was a resident at the Massachusetts General Hospital, where he was involved in designing and

implementing a point-of-care quality assurance programme. He has published a number of articles and chapters in the area of point-ofcare testing and is regularly invited

to speak on the management of point-of-care technologies. In 2002, Dr Lewandrowski founded a new journal, Point-of-Care, and is its Editor-in-Chief. He was trained in anatomic and clinical pathology at the Massachusetts General Hospital.
Point-of-care testing (POCT) is defined as laboratory testing that is performed at or near the site of patient care. The principal justification for POCT is that rapid turnaround for patient test results can be achieved, facilitating medical decisions and improving the quality of care. Familiar POC tests include blood glucose, pregnancy tests and urine dipsticks. From these humble beginnings, POCT has emerged, over the past decade, to comprise nearly $10 \%$ of the US $\$ 30$ billion worldwide clinical diagnostics market. POC tests are available for an ever-increasing variety of analytes, including cardiac markers, blood gases, electrolytes, $\mathrm{HIV}$, influenza $\mathrm{A} / \mathrm{B}$ and anticoagulation testing. Moreover, POC tests and testing devices are becoming more sophisticated, with improved accuracy, precision and ease of use. One impetus for the recent increase in the use of POCT has been the need for healthcare systems to improve patient management and operational efficiency due to cost containment, bed capacity utilisation and reimbursement considerations.

Patient safety initiatives have identified medical delivery systems as the root cause of most medical errors. POCT processes involve multiple manual data entry steps, which introduces opportunities for error. Automatic identification technologies have proven to be useful tools for reducing medical error and improving patient safety. ${ }^{1}$ Bar codes have been the mainstay of automatic identification technology for the past 30 years. Unfortunately, healthcare systems have been one of the last settings to adopt bar codes. For example, only recently has bar coding been applied to medication administration systems to improve patient safety. In controlled settings, barcode-enabled medication administration systems have been demonstrated to dramatically reduce medication administration errors. ${ }^{2}$

Implementing Bar Coding at the POC

For bar coding to be successfully implemented for a POC test, all of the items to be identified to the system must be bar-coded, including the patient, operator, test strips and quality control (QC) materials. The POC diagnostics industry has embraced bar coding, and many POC devices now have a built-in bar code scanner to improve data entry. Bar coding of test strips and quality control (QC) materials has simplified the process for entry of this critical data into the system. However, to 'close the loop' in the system, the two individuals in the process, the test operator and the patient, need to be bar-coded as well. For the test operator, his/her unique identity (ID) may be already bar-coded on the hospital ID badge. For hospitals that lack operator bar codes on their ID badges, a simple solution is to place a sticker with the bar-coded operator identifier on the back of the operator's badge. Rapid, accurate and automated identification of the patient remains a challenge in the POCT process. In the majority of healthcare institutions, the patient wristband does not contain a bar code, creating a situation where the patient ID must be manually typed into the POC device.

In practice, however, bar codes have several disadvantages that have contributed to their slow adoption in the healthcare environment. First, bar codes can be difficult to read in the harsh environments common in healthcare (due to blood stains, moisture and wear and tear). Second, the number of characters that can be encoded is limited (particularly in the case of linear or one-dimensional bar codes). Third, bar codes must be read in a line-of-sight manner, requiring careful alignment of the bar code with the reader. Finally, bar codes are not read-writable and cannot be updated on either a continuous or an intermittent basis. The limitations of bar coding technology have led to an interest in alternative systems for automatic identification in healthcare settings.

\section{Radiofrequency Identification}

A technology that does not suffer from these limitations is radiofrequency identification (RFID).

1. Bates $D W$, Cohen $M$, Leape $L L$ et al., "Reducing the frequency of errors in medicine using information technology", J. Am. Med. Inform. Assoc. (2001);8: pp. 299-308.

2. Patterson E S, Rogers $M L$, Render $M L$, "Fifteen best practice recommendations for bar-code medication administration in the Veterans Health Administration”, Jt Comm. J. Qual. Saf. (2004);30: pp. 355-365. 


\begin{tabular}{lllllll} 
& Ruggedness & Data Capacity & $\begin{array}{l}\text { Updateable } \\
\text { Dependence }\end{array}$ & $\begin{array}{l}\text { Readable Through } \\
\text { Other Objects }\end{array}$ & $\begin{array}{l}\text { Simultaneous Scanning } \\
\text { of Multiple Codes/tags }\end{array}$ & Orientation \\
\hline Bar code & No & $\begin{array}{l}\text { Limited }(<20 \\
\text { characters for } \\
\text { linear bar codes) }\end{array}$ & No & No & No & Yes \\
\hline RFID tag & Yes & $\begin{array}{l}\text { Thousands } \\
\text { of characters }\end{array}$ & Yes & Yes & Yes & No \\
\hline
\end{tabular}

This technology, while new and still evolving, offers several advantages over other automatic identification technologies (see Table 1). RFID is being investigated as an alternative to bar codes in many industries. RFID systems use a small electronic tag, consisting of a small integrated circuit attached to a miniature antenna, that is read by an RFID scanner analogous to the way that a bar code reader reads a printed bar code. Data about the tagged object is stored in the memory of the integrated circuit and information is sent wirelessly to the reader from the RFID tag via radiofrequency signals (see Figure 1). Today, RFID is used in a wide variety of applications, including automatic toll collection, wireless pay-at-the-pump programmes, electronic article surveillance tags and access control cards.

Using RFID tags rather than bar codes has significant advantages. Bar codes are typically applied to objects via paper labels and are, thus, susceptible to being torn, smudged or becoming detached from their objects. RFID tags are rugged, can be embedded in the material of the object itself and are readable even when the tag is dirty and at extreme temperatures. In addition, RFID tags can be read through many materials, so a direct line of sight with the RFID reader is not required. Furthermore, an RFID reader can scan multiple RFID tags simultaneously, in contrast to the bar code process in which each barcoded item must be individually oriented before scanning. Finally, linear bar codes have a practical storage capacity of only 10-20 characters, while RFID tags are less limited in their storage capacity and may store thousands of characters of data. RFID scanners have significant advantages over bar code readers. RFID scanners have no moving parts and may prove more reliable than bar code readers, requiring essentially no maintenance. The current efforts towards miniaturisation and standardisation of RFID scanners will permit the incorporation of such scanners into even the smallest of POCT devices.

\section{POC and RFID}

RFID capability has not yet been embraced by the POC diagnostics industry. Reagents and test
Figure I: How a radiofrequency identification (RFID) tag works,
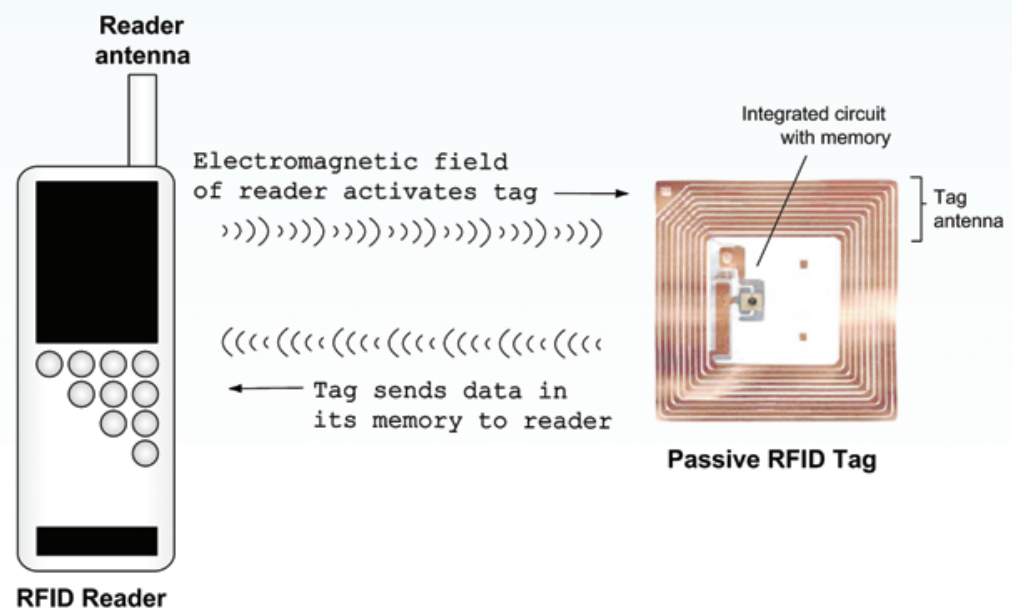

Passive RFID Tag

Passive (no battery) RFID tags work by having a RFID reader transmit a radiofrequency energy field that interacts with the antenna on the tag to create current for the integrated circuit, "waking up" the chip. Once activated, the tag accesses its stored data and then utilizes its built in antenna to exchange information with the reader.

Figure 2. Point of care testing in a radiofrequency enabled environment.

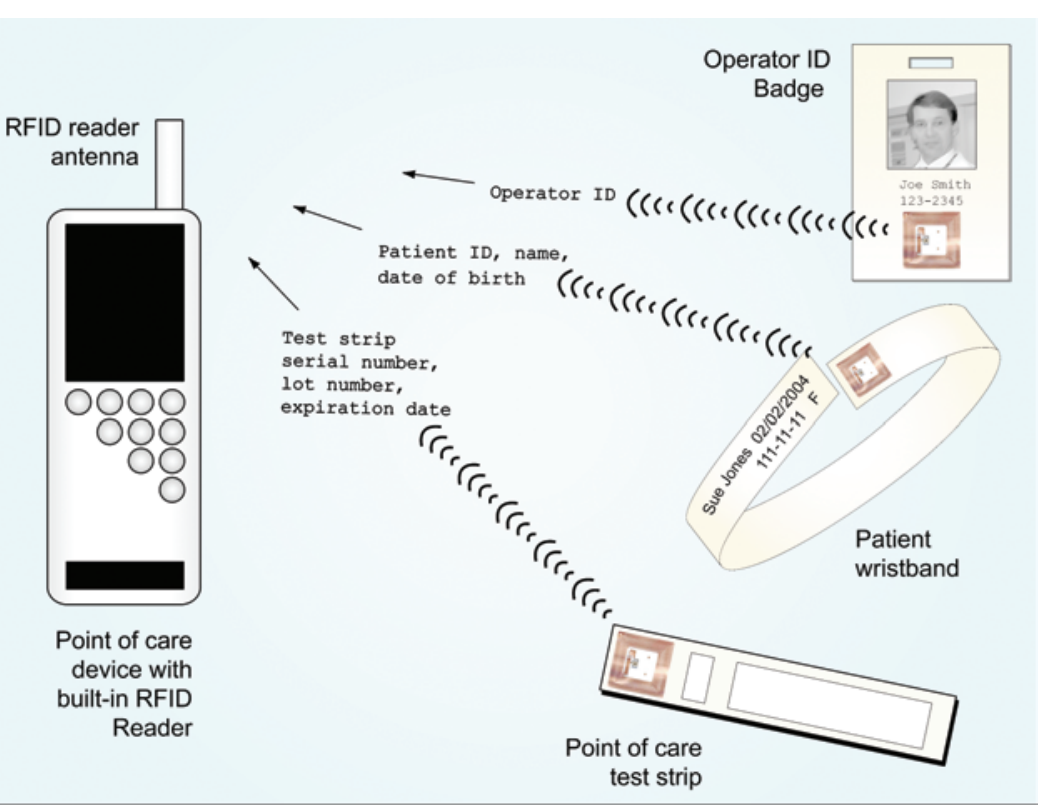

cartridges will need to be fitted with RFID tags, and hand-held POCT devices will require built-in RFID readers to enable RFID technologies to be used. However, as this technology is adopted by industry and the consumer products marketplace, it is likely that RFID readers and tags will be 
simplified and become less expensive and, thus, can be included in the design of systems without undue burden to manufacturers.

With full use of RFID technologies, the process of POCT could be simplified considerably (see Figure 2). A POCT device with a built-in RFID reader could gather information from all RFID-tagged objects within its reading range to quickly document each of the data elements necessary to create an error-free, medically valid POC test result. RFID tags on test strips/cartridges, operator ID badges, patient wristbands and QCs would make the entire documentation process essentially automatic, with an error rate approaching zero. Integrating automated information-gathering with POC electronic data management systems could provide alerting and interpretive information and assist in reducing errors in POCT processes. For example, the device could automatically recognise out-of-date or incompatible reagents or test cartridges and alert the user to their presence. Reducing operator dependence on documentation may also permit increasingly sophisticated tests to be developed as POC tests.

\section{Conclusions}

Automatic identification technologies such as bar coding and RFID have the potential to dramatically improve the documentation and identification process for POCT, particularly for healthcare in general. It has been asserted that "RFID is the key to automating everything", ${ }^{3}$ because RFID is an enabler in a networked world where each object has its own RFID tag and can interact with surrounding objects via RFID technology. The POC industry has grown rapidly over the past decade. Embracing RFID and other wireless technologies will help sustain this growth and streamline the process of POCT, reducing errors and simplifying training and documentation. 